\title{
NONLINEAR EVOLUTION EQUATIONS IN BANACH LATTICES
}

\author{
BY BRUCE CALVERT
}

Communicated February 27, 1970

1. Nonlinear operators in a Banach lattice. We recall a Banach lattice is a Banach space $X$ over the real numbers $R$, which is a lattice under the ordering $\leqq$, satisfying for $x, y, z$ in $X$ and $a \geqq 0$ in $R$,

(1) $x \leqq y$ implies $x+z \leqq y+z$,

(2) $x \leqq y$ implies $a x \leqq a y$, and

(3) $|x| \leqq|y|$ implies $\|x\| \leqq\|y\|$.

Following [12] we write $x^{+}=\sup (x, 0)$ and $x^{-}=\sup (-x, 0)$, giving $x=x^{+}-x^{-}$and $|x|=x^{+}+x^{-}$. A positive duality map $J$ is a function from $X$ to the dual $X^{*}$ with

(1) $(J x, x)=\|x\|^{2}$,

(2) $\|J x\|=\|x\|$,

(3) $(J x, y) \geqq 0$ if $x \geqq 0$ and $y \geqq 0$, and

(4) $(J x, y)=0$ if $x \perp y$ (i.e. $\inf (|x|,|y|)=0)$.

This was introduced in [10].

Proposition 1.1. A Banach lattice has a positive duality map.

If $g$ is a convex real valued function on $X$, then the subgradient $\operatorname{dg}: X \rightarrow$ subsets of $X^{*}$ is defined by: $w$ is a $\operatorname{dg}(x)$ iff for all $u$ in $X$, $g(u) \geqq g(x)+(w, u-x)$. A selection of a function $F: X \rightarrow$ subsets of $Y$ is a function $f: X \rightarrow Y$ with $f(x)$ in $F(x)$ for $x$ in $X$.

Proposition 1.2. If $X$ is a Banach lattice with positive duality map $J$ then $y \rightarrow 2 J\left(y^{+}\right)$is a selection of the subgradient of $y \rightarrow\left\|y^{+}\right\|^{2}$.

In the following we study existence of properties of solutions $x(t)$, $t \geqq 0$, of the equation of evolution

$$
d x / d t(t)=-A x(t), \quad x(0)=x_{0}
$$

for a given element $x_{0}$ of $D(A) \subset X$, where $A: D(A) \rightarrow X$ is a nonlinear operator (i.e. a function). In $\S \S 1$ and 2 , the theory is similar to [3], [4], [5], [7], [8], but is in the Banach lattice setting of [10], [11]. Important properties of $A$ are as follows. See [1] for the similar concept of a $T$-monotone operator.

AMS Subject Classifications. Primary 4720, 4750, 4780.

Key Words and Phrases. Banach lattice, nonlinear equations of evolution, duality map, subgradient, monotonic, $T$-accretive, $T$-nonexpansive, semigroup, fixed point, ergodic. 
Definition. Suppose $X$ a Banach lattice with positive duality map J. $A: D(A) \rightarrow X$ is:

(a) $T$-accretive if $\left(A x-A y, J\left((x-y)^{+}\right)\right) \geqq 0$ for $x, y$ in $D(A)$,

(b) generalised $T$-accretive if there exists $k$ in $R$ with

$$
\left(A x-A y, J\left((x-y)^{+}\right)\right) \geqq-k\left\|(x-y)^{+}\right\|^{2} \quad \text { for } x, y \text { in } D(A),
$$

(c) locally generalised $T$-accretive if for $z$ in $D(A)$ there is $k_{z}$, in $R$ and a neighborhood $N_{z}$ of $z$ in $X$, with

$\left(A x-A y, J\left((x-y)^{+}\right)\right) \geqq-k_{z}\left\|(x-y)^{+}\right\|^{2} \quad$ for $x, y$ in $N_{z} \cap D(A)$.

The importance of these definitions, and motivations, is the following. Supposing solutions to the equation of evolution exist uniquely, for $t \geqq 0$ we have a function $U(t)$ taking $x_{0}$ to $x(t)$. Then we have $U(t)$ is a monotonic function, and if $A x_{0} \leqq 0$ then $x(t)$ is increasing. We recall [9] a function $U: D(U) \rightarrow X$ is monotonic if $x \leqq y, x, y$ in $D(U)$ implies $U(x) \leqq U(y)$.

Definition. Supposing $X$ is a Banach lattice, then $U: D(U) \rightarrow X$ is:

(a) T-nonexpansive if $\|(U x-U y)+\| \leqq\|(x-y)+\|$ for $x, y$ in $D(U)$,

(b) T-Lipschitz if there is $k$ in $R$ with $\|(U x-U y)+\| \leqq k\|(x-y)+\|$ for $x, y$ in $D(U)$,

(c) locally $T$-Lipschitz if, for $z$ in $D(U)$, there is a neighborhood $N_{z}$ of $z$ in $X$, and $k_{z}$ in $R$, with $\|(U x-U y)+\| \leqq k_{z}\|(x-y)+\|$ for $x, y$ in $N_{z} \cap D(U)$.

Proposition 1.3. A locally T-Lipschitz function $U$ with convex domain is monotonic. $A C^{1}$ function $U$ with open domain is locally $T$ Lipschitz.

Proposition 1.4. Suppose $X$ a Banach lattice with positive duality map. If $U: D(U) \rightarrow X$ is $T$-nonexpansive, then $I-U$ is T-accretive. If $A: D(A) \rightarrow X$ is $T$-accretive, then for all $d>0,(I+d A)^{-1}: R(I+d A) \rightarrow X$ is $T$-nonexpansive, and conversely if $J$ is continuous from the strong to the weak* topology.

Proposition 1.5. Suppose $X$ a Banach lattice with positive duality map, and $A$ a hypermaximal T-accretive function, i.e. $A: D(A) \rightarrow X$ is $T$-accretive and $R(I+A)=X$. Then $R(I+d A)=X$ for all $d>0$, and $A(I+d A)^{-1}$ is $T$-accretive and Lipschitzian from $X$ to $X$.

Theorem 1.6. Suppose $X$ is a Banach lattice with positive duality map. Suppose $A: D(A) \rightarrow X$ is a function such that for $x_{0}$ in $D(A)$ there are strongly continuous weakly once differentiable solutions to $d x / d t(t)$ $=-A x(t)$, with initial condition $x(0)=x_{0}$, for $t$ in an interval $[0, h)$. For $t \geqq 0$ we say $x$ is in $D(U(t))$ if $h>t$, and set $U(t) x_{0}=\{x(t): x a$ 
solution as above $\}$. Then for $t \geqq 0, U(t)$ is: (a) a T-nonexpansive function, (b) a T-Lipschitz function or (c) a locally T-Lipschitz function, iff $A$ is (a) T-accretive, (b) generalised T-accretive or (c) locally generalised $T$-accretive.

In this case, we have

(a) $\|(A x(t))+\| \leqq\left\|\left(A x_{0}\right)+\right\|$,

(b) $\|(A x(t))+\| \leqq e^{k t}\left\|\left(A x_{0}\right)+\right\|, \quad$ where $\left(A x-A y, J\left((x-y)^{+}\right)\right)$ $\geqq-k\|(x-y)+\|^{2}$, or

(c) $\left\|(A x(t))^{+}\right\| \leqq e^{K(t)}\left\|\left(A x_{0}\right)^{+}\right\| \quad$ where $\left(A x-A y, J\left((x-y)^{+}\right)\right)$ $\geqq-k(y)\left\|(x-y)^{+}\right\|^{2}$ for $x$ near $y$ and $K(t)=\int_{0}^{t} k(x(s)) d s$.

We recall [3] that $U: D(U) \rightarrow X$ is nonexpansive if $\|U x-U y\|$ $\leqq\|x-y\|$ for $x, y$ in $D(U)$. A Banach lattice has property $P$ [2] if $a, b, c, d \geqq 0, a \perp b, c \perp d,\|a\|=\|c\|$, and $\|b\|=\|d\|$ implies $\|a+b\|$ $=\|c+d\|$.

Proposition 1.7. Any Banach lattice has an equivalent norm in which $T$-nonexpansive functions are nonexpansive. Every T-nonexpansive function $U: D(U) \rightarrow X$ is nonexpansive iff $X$ has property $P$.

Proposition 1.8. Suppose $X$ an AL space with positive duality map (i.e., $x \geqq 0, y \geqq 0$ implies $\|x+y\|=\|x\|+\|y\|$ ). The fixed point set $F(U)$ of a T-nonexpansive function $U: X \rightarrow X$ is a sublattice of $X$. If $A: D(A) \rightarrow X$ is hypermaximal $T$-accretive then $A^{-1}(x)$ is a sublattice for $x$ in $X$.

2. Existence of solution to equations of evolution. We recall a Banach space $Y$ is uniformly convex if for $e>0$ there exists $d>0$ such that $\|x\| \leqq 1,\|y\| \leqq 1,\|x+y\| \geqq 2-d$, implies $\|x-y\|<e$. We say a function $A$ is demicontinuous if it is continuous from the strong to the weak topology.

Theorem 2.1. Suppose $X$ a Banach lattice with $X^{*}$ uniformly convex. Suppose $G$ open in $X$ and $A_{1}: G \rightarrow X$ is demicontinuous and locally generalised T-accretive. Suppose $A_{2}: D\left(A_{2}\right) \rightarrow X$ is hypermaximal $T$ accretive. Let $A=A_{1}+A_{2}, D(A)=D\left(A_{2}\right) \cap G$. For $x_{0}$ in $D(A)$ there is an interval $[0, d]$ and a unique continuous weakly $C^{1}$ function $x:[0, d] \rightarrow X$ with $x(0)=x_{0}$ and $d x / d t(t)=-A x(t)$. The strong derivative of $x$ exists almost everywhere and equals $-A x(t)$.

Theorem 2.2. Suppose $X$ a Banach lattice with positive duality map. Suppose $G$ open in $X$ and $A: G \rightarrow X$ is locally generalised T-accretive and locally uniformly continuous (each point of $G$ has a neighborhood on which $A$ is uniformly continuous). Then for $x_{0}$ in $G$ there is an interval $[0, d]$ and a unique strongly $C^{1}$ function $x:[0, d] \rightarrow X$ with $x(0)=x_{0}$ and $d x / d t=-A x(t)$. 
Theorem 2.3. Suppose $X$ a Banach lattice with $X^{*}$ uniformly convex, $A_{1}: X \rightarrow X$ is demicontinuous and $T$-accretive, $A_{2}: D\left(A_{2}\right) \rightarrow X$ and $A_{3}: D\left(A_{3}\right) \rightarrow X$ are hypermaximal T-accretive, with $D\left(A_{2}\right) \subset D\left(A_{3}\right)$. Suppose for $x$ in $X$ there is a neighborhood $N_{x}$ of $x, k_{x}<1, h_{x}$ in $R$, with $\left\|A_{z} y\right\| \leqq k_{x}\left\|A_{z} y\right\|+h_{x}$ for $y$ in $D\left(A_{2}\right) \cap N_{x}$. Then $A=A_{1}+A_{2}+A_{3}$ is hypermaximal $T$-accretive.

\section{The range of $A$.}

THEOREM 3.1. Let $X$ be a Banach lattice with $X^{*}$ uniformly convex. Let $A_{1}: D\left(A_{1}\right) \rightarrow X$ be hypermaximal $T$-accretive. Let $A_{2}: X \rightarrow X$ be demicontinuous and locally generalised $T$-accretive. Let $A=A_{1}+A_{2}$; $D(A)=D\left(A_{1}\right)$.

Suppose either (a) for $a, b$ in $X\{x: a \leqq x$ and $A x \leqq b\}$ and $\{x: a \geqq x$ and $A x \geqq b\}$ are bounded, or

(b) $A$ is $T$-accretive outside a bounded set and $A^{-1}$ is bounded.

Then $A$ is surjective, and $A^{-1}$ has a monotonic selection. Furthermore, if there exist $x, y$ with $A x \leqq x \leqq y \leqq A y$, then there is a fixed point of $A$ in $[x, y]$.

THEOREM 3.2. Let $X$ be a Banach lattice with positive duality map. Suppose $X$ is fully regular, i.e. any bounded set directed under $\leqq$ is convergent [9].

Suppose $A: D(A) \rightarrow X$ is hypermaximal $T$-accretive, and $A^{-1}$ is locally bounded. Then $A$ is surjective, and $A^{-1}$ is monotonic if it is single valued and demicontinuous.

Theorem 3.3. Suppose $X$ an order complete Banach lattice with positive duality map (i.e. if $A \subset X$ is order bounded then $\sup (A)$ and $\inf (A)$ exist). ( $A$ is order bounded means it is contained in an order interval $[a, b]=\{x$ in $X: a \leqq x \leqq b\}$.) Suppose $A: X \rightarrow X$ is locally uniformly continuous and locally generalised $T$-accretive. Then for $a \leqq b$ in $X,[A(a), A(b)] \subset A[a, b]$.

TheOREM 3.4. Suppose $X$ an order complete Banach lattice with positive duality map whose positive cone $\{x$ in $X: x \geqq 0\}$ has nonempty interior. Suppose $A: D(A) \rightarrow X$ is hypermaximal $T$-accretive and $A^{-1}$ is locally bounded. Then $A$ is surjective.

Theorem 3.5. Suppose $G$ a closed bounded convex subset of a reflexive Banach lattice $X$. Let $B_{e}(G)=\{x$ in $X: d(x, G) \leqq e\}$. Suppose $U: B_{e}(G)$ $\rightarrow X$ is locally $T$-Lipschitz with $U x$ in $G$ if $d(x, G)=e$. Then $(1-U) B_{0}(G)$ is closed. 
Corollary. Suppose $U$ as above is T-nonexpansive. Then $U$ has a fixed point in $G$.

\section{Ergodic theory.}

Theorem 4.1. Suppose $X$ is a uniformly convex Banach lattice with positive cone $K$. Suppose $U: K \rightarrow K$ is nonlinear, and $U x \leqq W x$ for $x$ in $K$, where $W$ is linear and $T$-nonexpansive. Then for $x$ in $K, S_{n} x$ $=n^{-1} \sum_{i=1}^{n} U^{i}(x)$ converges to $x_{0}$ in $K$. The function $S_{0}: K \rightarrow K$ taking $x$ to $x_{0}$ satisfies $S_{0} U x=S_{0} x \geqq U S_{0} x \geqq S_{0}^{2} x=S_{0}^{n} x, n \geqq 2$, if $U$ is continuous and monotonic, and the range of $S_{0}^{2}$ is the fixed point set of $U$.

Theorem 4.2. Suppose $X$ is a Banach lattice with $X$ and $X^{*}$ uniformly convex. Suppose $A: D(A) \rightarrow X$ is the sum of a hypermaximal $T$ accretive and a demicontinuous generalised $T$-accretive function, and $A(0)=0$.

Suppose $B: D(B) \rightarrow X$ is linear and hypermaximal $T$-accretive. Suppose $D(B) \subset D(A)$. Suppose for in $K \cap D(B)$ we have $A x \geqq B x$. For $x_{0}$ in $D(A)$, define $U(t) x_{0}=x(t)$ for $t \geqq 0$, where $x(0)=x_{0},(d x / d t) \quad(t)$ $=-A x(t)$, and extend $U(t)$ by continuity to $K$. Then for $z$ in $K$, $S_{t}(z)=t^{-1} \int_{0}^{t} U(t) z$ converges to $z_{0}$ in $K \cap D(A)$, with $A\left(z_{0}\right) \geqq 0$.

5. Some further developments. The author has developed some results for monotonic generators, and also obtained results for $X$ an algebra. The following are examples.

Theorem 5.1. Suppose $X$ an order complete Banach lattice. Suppose $B: G \rightarrow X$ is monotonic and continuous, $G$ open in $X$. Suppose each point has a neighborhood $N$ with $B(N)$ order bounded. Then for $x_{0}$ in $G$ there is an interval $[0, d]$ and a strongly $C^{1}$ function $x:[0, d] \rightarrow X$ with $x(0)=x_{0}$ and $(d x / d t)(t)=B x(t)$.

Theorem 5.2. Suppose $X$ is the dual of an $A L$ space. Suppose $T: D(T) \rightarrow X$ satisfies $R(1+T)=X$ and $(T x-T y)(x-y) \geqq 0$ for $x, y$ in $D(T)$ (cf. [6]). Then for $x_{0}$ in $D(T)$ there exists a unique continuous weak $C^{1}$ function $x:[0, \infty) \rightarrow X$, with $x(0)=x_{0}$, and $(d x / d t)(t)=-T x(t)$.

These results will appear with proofs elsewhere. The author is very grateful to Professor Felix Browder for introducing him to semigroups of nonlinear operators, and to Dr. Peter Hess for help with presentation of this work as part of a thesis.

\section{BIBLIOGRAPHY}

1. H. Brezis and G. Stampacchia, Sur la régularite de la solution d'inéquations elliptiques, Bull. Soc. Math. France 96 (1968), 153-180. MR 39 \#659. 
2. F. Bohnenblust, An Axiomatic characterization of $L_{p}$-spaces, Duke Math J. 6 (1940), 627-640. MR 2, 102.

3. F. Browder, Nonlinear operators and nonlinear equations of evolution in Banach spaces, Proc. Sympos. Pure Math., vol. 18, Part II, Amer. Math. Soc., Providence, R.I., (to appear).

4. M. Crandall and A. Pazy, Semigroups of nonlinear contractions and dissipative sets, J. Functional Analysis 3(1969), 376-418.

5. J. Dorroh, $A$ nonlinear Hille-Yosida-Phillips theorem, J. Functional Analysis 3 (1969), 345-353. MR 39 \#2019.

6. R. Kacurovskil, Three theorems on nonlinear equations involving monotone operators, Dokl. Akad. Nauk SSSR 183 (1969), 33-36=Soviet Math. Dokl. 9 (1968), 13221325.

7. T. Kato, Nonlinear semigroups and evolution equations, J. Math. Soc. Japan 19 (1967), 508-520. MR 37 \#1820.

8. Y. Kömura, Nonlinear semi-groups in Hilbert space, J. Math. Soc. Japan 19 (1967), 493-507. MR $35 \# 7176$.

9. M. Krasnosel'ski1, Positive solutions of operator equations, Fizmatgiz, Moscow, 1962; English transl., Noordhoff, Groningen, 1964. MR 26 \#2862.

10. R. Phillips, Semi-groups of positive coniraction operators, Czechoslovak Math. J. 12 (87) (1962), 294-313. MR 26 \#4195.

11 . K. Sato, On the generators of non-negative contraction semi-groups in Banach lattices, J. Math. Soc. Japan 20 (1968), 423-436. MR 37 \#6798.

12. H. Schaefer, Topological vector spaces, Macmillan, New York, 1966. MR 33 \#1689.

Universtty of Colorado, Boulder, Colorado 80302 